\title{
Riqueza de espécies de morcegos (Mammalia, Chiroptera) em dois diferentes habitats, na região centro-sul do Paraná, sul do Brasil
}

\author{
Nelio R. dos Reis ${ }^{1}$; Adriano L. Peracchi ${ }^{2}$; Isaac P. de Lima ${ }^{2} \&$ Wagner A. Pedro $^{3}$ \\ ${ }^{1}$ Departamento de Biologia Animal e Vegetal, Universidade Estadual de Londrina. Caixa Postal 6001, 86051-970 Londrina, \\ Paraná, Brasil. \\ ${ }^{2}$ Laboratório de Mastozoologia, Instituto de Biologia, Universidade Federal Rural do Rio de Janeiro. Caixa Postal 74503, \\ 23851-970 Seropédica, Rio de Janeiro, Brasil. \\ ${ }^{3}$ Departamento de Apoio à Saúde e Produção Animal, Universidade Estadual Paulista Julio de Mesquita Filho. \\ 16050-680 Araçatuba, São Paulo, Brasil.
}

\begin{abstract}
Richness of bats species (Mammalia, Chiroptera) in two different habitats in the center south region, Paraná, Brazil. Preliminary study about the richness of species and the capture frequency of bats from two habitats: native forest and Araucária reforestation area, in the center east region of Paraná. The same capture effort for the two areas resulted in 11 species of bats captured in the native forest and eight in the Araucária reforestation area. The uniformity in species abundance - evenness - was higher in the native forest. In the Araucária reforestation area, ninety per cent of the collected individuals belonged only to two species. Results show that the primary forest with numerous niches presents high richness of species, but human action reduces the general richness, which reflects mainly in the small number of species. Myotis ruber is registered in both environments and related in the Brazilian list of threatened species of extinction.
\end{abstract}

KEY WORDS. Animal diversity; ecologic park; forest fragments.

RESUMO. Estudo preliminar sobre a riqueza de espécies e a frequiência de capturas de morcegos em dois habitats, mata primária e reflorestamento de Araucária, na Região Centro Leste do Paraná. Foi empregado o mesmo esforço para as duas áreas, resultando na captura de 11 espécies na área de mata primária, contra oito no reflorestamento de Araucária. A uniformidade na distribuição da abundância - "equitabilidade" - foi maior na mata primária. No reflorestamento de Araucária, noventa por cento dos indivíduos coletados pertenciam somente a duas espécies. Pelos resultados, conclui-se que a floresta primária com numerosos nichos, apresenta uma riqueza de espécies elevada, mas a ação do homem reduz a riqueza de espécies geral, o que se reflete principalmente na dominância de poucas espécies. Destaca-se aqui, nos dois tipos de ambientes, a presença de Myotis ruber, listada como ameaçada de extinção no Brasil.

PALAVRAS-CHAVE. Diversidade de morcegos; fragmentos florestais; parque ecológico.

O ambiente sofre variações no tempo e no espaço. Nenhum organismo pode manipular igualmente e eficientemente as condições físicas, os predadores e presas, em todos os tipos de habitats (Ricklefs 1993). Desse modo, os ambientes heterogêneos, requerem dos seus indivíduos escolhas concernentes ao uso do habitat, como a seleção do micro-habitat, de presas, entre outros. Segundo PIANKa (1982), estas escolhas visam otimizar as relações das espécies em cada local.

O solo, a topografia e o clima influenciam os vários ambientes terrestres, determinando que tipo de vegetação irá se estabelecer e que tipo de composição faunística terá suporte neste ambiente. As comunidades biológicas de diferentes áreas apresentam diferentes fisionomias que permitem a ocorrência de vá- rias espécies de mamíferos. À medida que muda o substrato, altera-se a vegetação, e a composição faunística associada, que está relacionada à oferta de alimentos, à qualidade dos abrigos, e às condições para reprodução, fatores esses que irão determinar o equilíbrio dinâmico da comunidade (REMMERT 1982, RICKLEFS 1993, WILSON 1997).

Um passo importante para verificar o estado de conservação de uma região, seria avaliar as populações de morcegos. Medellin et al. (2000), encontrou uma significativa associação entre baixos níveis de perturbação e um número alto de espécie de phyllostomideos num trabalho realizado com cinco hábitats em Chiapas, México.

Baseados nessas informações avaliamos o número de es- 


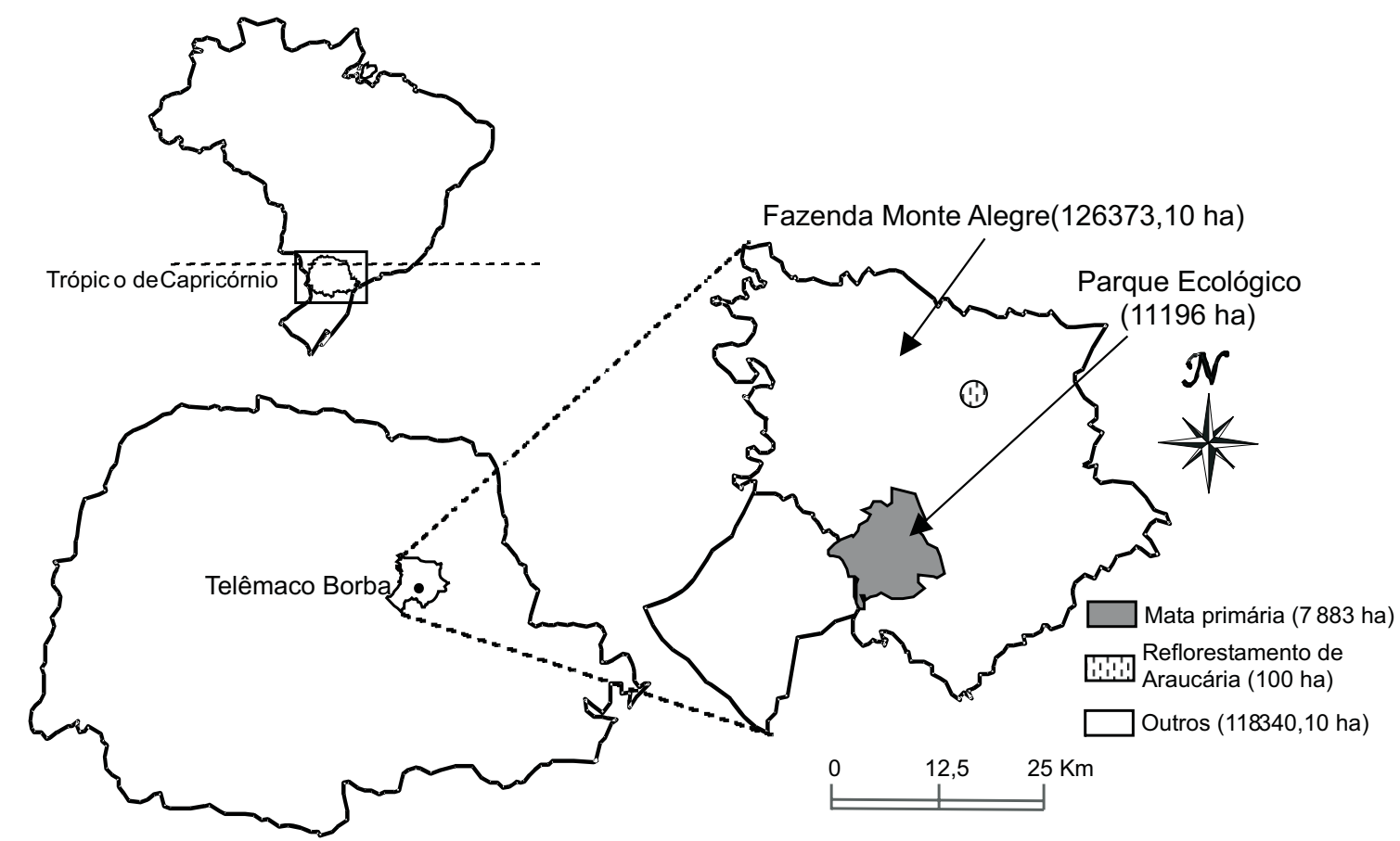

Figura 1. Localização do município de Telêmaco Borba, da Fazenda Monte Alegre e do Parque Ecológico da Klabin e os pontos onde foram realizadas as coletas.

pécies e o número de indivíduos de morcegos em dois ambientes: um em mata primária e outro, em um reflorestamento de Araucária na região Centro Sul do Paraná.

Estudos relacionados à riqueza de espécies e à da fauna de quirópteros em fragmentos florestais já foram realizados no Paraná por Reis \& Muller (1995), Reis et al. (1998, 1999, 2000, 2003), Félix et al. (2001).

\section{MATERIAL E MÉTODOS}

Tanto a mata primária quanto a área de reflorestamento estudadas estão dentro dos limites da Fazenda Monte Alegre (Fig. 1) que possui $126.373,10$ ha e está localizada nas seguintes coordenadas geográficas $24^{\circ} 12^{\prime} 42^{\prime \prime} \mathrm{S}$ e $50^{\circ} 33^{\prime} 26^{\prime \prime} \mathrm{W}$, dentro do município de Telêmaco Borba. A altitude média é de $885 \mathrm{~m}$ e o clima, segundo a classificação de Köppen (Trewartha \& Horn 1980), enquadra-se como subtropical com a temperatura média do mês mais frio de $16,3^{\circ} \mathrm{C}$ e a temperatura média do mês mais quente $23,2^{\circ} \mathrm{C}$. A precipitação pluviométrica média anual é de $1478 \mathrm{~mm}$.

A mata primária estudada (7883 ha) está nos limites do Parque Ecológico da Klabin (11196 ha), sendo constituída por florestas naturais do tipo ombrófila mista, algumas delas ainda intactas, com árvores altas, destacando-se excepcionais exemplares de Araucaria angustifolia (Bert.) O. Ktze (pinheiro-doparaná), Patagonuta americana L. (guajavira) e Eugenia envolucrata DC. (cerejeira). O sub-bosque é dominado por samambaias, bromélias, cipós e avencas.
O reflorestamento de Araucária está a 30 km de distância da área de mata primária, porém dentro dos limites da Fazenda, sendo composto por vários reflorestamentos de pinheirosdo-paraná, com idade aproximada de 30 anos, com um subbosque formado principalmente por taquaras. A área total do reflorestamento de araucária soma 100 ha, que somados aos outros ao redor alcançam aproximadamente 10000 ha.

A amostragem consistiu em quatro coletas mensais, sendo duas em mata nativa e duas em área de reflorestamento. As redes foram armadas com o mesmo esforço de captura nos dois tipos de ambiente, em lugares preferidos pelos morcegos como: trilhas, estradas abandonadas e sobre riachos de acordo com Greenhall \& Paradiso (1968). Para cada tipo de ambiente foram utilizadas oito redes do tipo "mist net" $(7$ x 2,5 m), distantes $50 \mathrm{~m}$ uma da outra, perfazendo $140 \mathrm{~m}^{2}$ de exposição em cada noite de coleta. As redes permaneciam estendidas por quatro horas após o pôr-do-sol, pois segundo MorRIson (1978) a atividade dos morcegos é maior nas primeiras horas da noite. Com oito horas mensais de redes estendidas em cada tipo de ambiente, temos um total de 128 horas de exposição ao final oito meses de trabalho, de março a outubro de 2004.

Os animais não identificados em campo foram encaminhados para o Laboratório de Ecologia da Universidade Estadual de Londrina para identificação, de acordo com os critérios de: Vieira (1942), Husson (1962), Vizotto \& TAddei (1973), Jones \& Carter (1976), Medelí́n et al. (1997). Somente dois exemplares de 

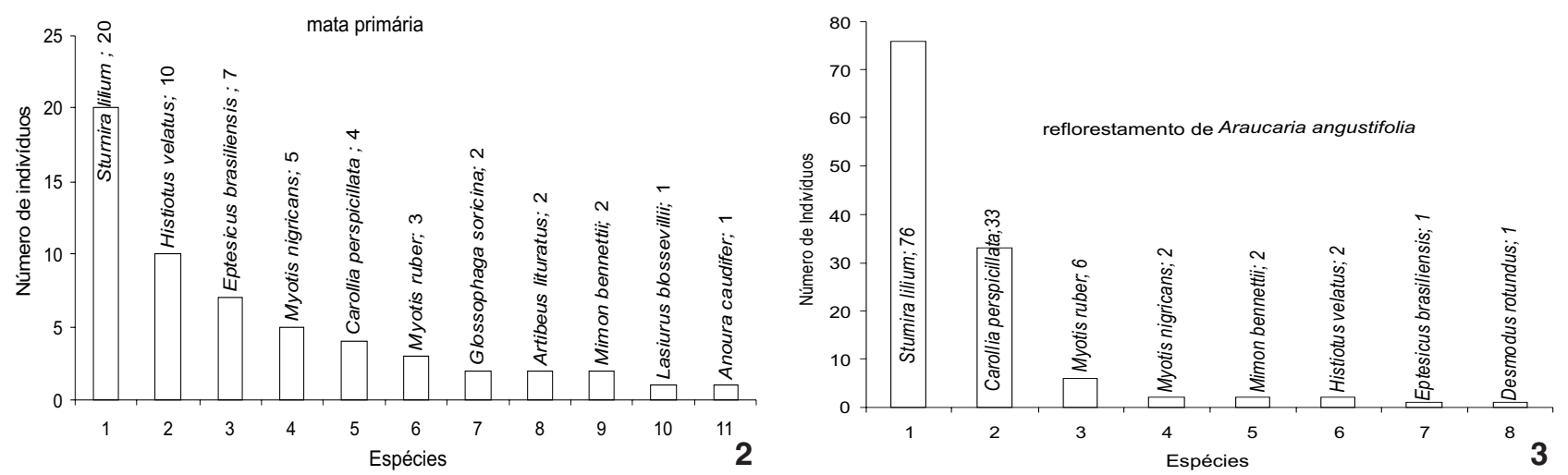

Figura 2. Espécies e indivíduos de morcegos amostrados em área de: (2) mata primária e (3) reflorestamento da Fazenda Monte Alegre, no período de março a outubro de 2004.

cada espécie foram sacrificados e fixados em formol a 10\% e conservados em álcool 70\%, sendo depositados no Museu de Zoologia, Universidade Estadual de Londrina, Paraná e na Universidade Federal Rural do Rio de Janeiro, Seropédica, Rio de Janeiro onde, nesta última, receberam a confirmação das identificações.

\section{RESULTADOS}

Foi encontrado um maior número de espécies na mata primária (11) (Fig. 2), área que também apresentou maior equitabilidade entre as abundâncias das espécies. Para a área de Araucária foram registradas oito espécies (Fig. 3), local no qual, distinguindo-se da mata primária, houve grande dominância de duas espécies, Sturnira lilium e Carollia perspicillata, perfazendo 90\% dos indivíduos.

Os trabalhos de campo possibilitaram a captura das seguintes espécies: Mimon bennettii (Gray, 1838); Anoura caudifer (E. Geoffroy, 1818); Glossophaga soricina (Pallas, 1766); Carollia perspicillata (Linnaeus, 1758); Artibeus lituratus (Olfers, 1818); Sturnira lilium (E. Geoffroy, 1810); Desmodus rotundus (E. Geoffroy, 1810); Eptesicus brasiliensis (Desmarest, 1819); Histiotus velatus (I. Geoffroy, 1824); Lasiurus blossevillii (Lesson \& Garnot, 1826); Myotis nigricans (Schinz, 1821) e Myotis ruber (E. Geoffroy, 1806) (Figs 2 e 3).

\section{DISCUSSÃO}

Uma floresta primária, constituída de árvores altas, destacando-se Araucaria angustifolia (Pinheiro-do-Paraná), Patagonula americana (guajavira) e Eugenia involucrata (cerejeira), com sub-bosque dominado por samambaias, bromélias, cipós e avencas. De acordo com Odum (1985), florestas desse tipo possuem numerosos nichos e apresenta uma riqueza de espécies elevada. Na Fazenda Monte Alegre, isso é evidente, onde encontramos $27 \%$ de espécies a mais na mata primária do que no reflorestamento de Araucária. (Figs 2 e 3). A estrutura da mata favorece uma maior riqueza e equitabilidade de espécies. G. soricina, A. caudifer e L. blossevillii, que só foram en- contradas na mata primária, são aparentemente mais dependentes dos recursos desta. A ausência das duas primeiras espécies pode estar relacionada a um mesmo fator, já que ambas fazem parte da guilda dos nectarívoros.

A ação humana parece causar um desequilíbrio nas relações quantitativas entre as espécies, e modificar todo o cenário de condições de vida e da disponibilidade de recursos. Em áreas perturbadas por desmatamentos geralmente se desenvolvem, nas clareiras resultantes desses processos, plantas pioneiras como solanáceas, cecropiáceas e piperáceas, o que pode explicar a grande dominância das espécies S. lilium (62\%) e C. perspicillata (27\%), na área de reflorestamento. Reis et al. (1999), ao trabalharem em trilhas de visitação do Parque Ecológico da Klabin, obtiveram grande dominância de A. lituratus (43\%), com S. lilium e C. perspicillata representando, respectivamente, apenas $8,9 \%$ e $3,9 \%$ das capturas. Estes resultados sugerem que estas três espécies de frugívoros podem se alternar na dominância em diferentes comunidades de morcegos, pelo menos no sul do Brasil.

De fundamental importância é a ocorrência, nos dois tipos de ambientes, da espécie Myotis ruber, apontada como ameaçada de extinção na lista oficial de espécies ameaçadas do Brasil (MACHADo et al. 2005). Nossos resultados apontam a possibilidade de adaptação da espécie a ambiente alterado por reflorestamento de A. angustifolia, embora a frequência de ocorrência da espécie seja baixa tanto na mata nativa, como na área de reflorestamento, o que justifica a continuidade de esforços de preservação para a espécie.

Dentro do apresentado, consideramos ser importante analisar os dados em primeira instância no equilíbrio das matas, e depois interpretar as alterações nas bordas, não de forma inversa. Nem todas as espécies que existiam na mata conseguem sobreviver nas bordas ou no ecossistema modificado, porque: 1) Ocorrendo o desmatamento, altera-se o microclima local, com elevação da temperatura no contorno da mata, que recebe mais luminosidade. As espécies de morcegos estenotérmicas não resistem; 2) aumentando a luminosidade, desenvol- 
ve-se outro tipo de vegetação que não serve como recurso para as espécies mais sensíveis e especializadas; 3 ) sem a proteção da floresta muitas espécies pouco adaptadas ao desflorestamento, ficam muito mais susceptíveis aos predadores; 4) condições de transição favorecem espécies oportunistas e generalistas como Artibeus lituratus, que ocupam os novos nichos disponíveis, chegando em alguns caso a representar mais de $80 \%$ das capturas nas áreas de borda. Neste trabalho, 90\% das espécies da mata alterada são representadas por S. lilium e C. perspicillata; 5) perdem-se os abrigos como ocos de árvores, proteção de diferentes folhas, entre outros.

\section{AGRADECIMENTOS}

À Klabin S.A. pelo financiamento do projeto; à Universidade Estadual de Londrina; à Fundação de Amparo à Pesquisa do Estado do Rio de Janeiro E-26/152.621/2005 pela concessão de Bolsa a I.P.L.; à Fundação de Amparo à Pesquisa do Estado de São Paulo 98/08940-0 e ao Conselho Nacional de Desenvolvimento Científico e Tecnológico 300845/98-7 (RN) pela concessão de auxílio e bolsa à W.A.P. (300845/98-7 RN) e A.L.P. (300265/80-8).

\section{REFERÊNCIAS BIBLIOGRÁFICAS}

Félix, J.S.; N.R. dos Reis; I.P. de Lima; E.F. Costa \& A.L. Peracchi. 2001. Is the area of the Arthur Thomas Park, with its 82.72 ha, sufficient to maintain viable chiropteran populations? Chiroptera Neotropical, Brasília, 4 (1-2): 129-133.

Greenhall, A.M. \& J.L. Paradiso. 1968. Bats and bat banding. Washington, Bureau of Sport Fisheries and Wild, 47p.

Husson, A.M. 1962. The bats of Suriname. Zoolgische Verhandelingen. Leiden, 58: 1-282.

Jones, J.K. \& D.C. CARTER. 1976. Annotated checklist with keys to subfamilies and genera. In: Biology of bats the New World family Phyllostomatidae. Part I. Special Publication Museum Texas Tech University, Huston, 10: 7-38.

Machado, A.B.M.; C.S. Martins \& G.M. Drummond. 2005. Lista da fauna brasileira ameaçada de extinção. Belo Horizonte, Fundação Biodiversitas, 157p.

Medellín, R.A.; H. Arita \& O. Sánchez-Hernández. 1997. Identificación de los murciélagos de México: claves de campo. México, Asociación Mexicana de Mastozoología, Publicaciones especiales no.2, 89p.

Recebido em 17.I.2006; aceito em 25.VIII.2006.
Medellín, R.A.; M. Equinua \& M.A. Amin. 2000. Bat diversity and abundance as indicators of disturbance in Neotropical Rainforests. Conservation Biology, Boston, 14 (6): 1666-1675.

Morrison, D.W. 1978. Lunar phobia in a neotropical fruit bat Artibeus jamaicensis (Chiroptera, Phyllostomidae). Animal Behaviour, London, 26 (3): 852-855

Odum, E.P. 1985. Ecologia. Rio de Janeiro, Interamericana, 434p. PiAnKa, E.R. 1982. Ecologia evolutiva. Barcelona, Omega, 365p. ReIs, N.R. Dos \& M.F. Muller. 1995. Bat diversity of forest and open areas in a subtropical region of south Brazil. Ecologia Austral, Córdoba, 5: 31-36.

Reis, N.R. dos; A.L. Peracchi; I.P. de Lima; M.L. Sekiama \& V.J. RoCHA. 1998. Updated list of the chiropterians of the city of Londrina, Paraná, Brazil. Chiroptera Neotropical, Brasília, 4 (2): 96-98.

Reis, N.R. dos; A.L. Peracchi \& M.L. Sekiama. 1999. Morcegos da fazenda Monte Alegre, Telêmaco Borba, Paraná (Mammalia, Chiroptera). Revista Brasileira de Zoologia, Curitiba, 16 (2): 501-505.

Reis, N.R. dos; A.L. Peracchi; M.L. Sekiama \& I.P. de Lima. 2000. Diversidade de morcegos (Chiroptera: Mammalia) em fragmentos florestais no estado do Paraná, Brasil. Revista Brasileira de Zoologia, Curitiba, 17 (3): 697-704.

Reis, N. R. Dos; M.L.S. Barbieri; I.P. de Lima \& A.L. Peracchi. 2003. O que é melhor para manter a riqueza de espécies de morcegos (Mammalia, Chiroptera): um fragmento florestal grande ou vários fragmentos de pequeno tamanho? Revista Brasileira de Zoologia, Curitiba, 20 (2): 225-230.

Remmert, H. 1982. Ecologia. São Paulo, EDUSP, 335p.

RickLEFs, R.E. 1993. A economia da natureza: um livro texto de ecologia básica. Rio de Janeiro, Guanabara Koogan, 470p.

Trewartha, G.T. \& L.H. Horn. 1980. An introduction to climate. New York, McGraw-Hill, $5^{\text {th }}$ ed., 416p.

VieIRA, C.O.C. 1942. Ensaio monográfico sobre os quirópteros do Brasil. Arquivos de Zoologia, São Paulo, 3 (8): 1-471.

VizotTo, L.D. \& V.A. TADDEI. 1973. Chave para determinação de quirópteros brasileiros. Revista da Faculdade de Filosofia Ciências e Letras São José do Rio Preto - Boletim de Ciências, São José do Rio Preto, 1: 1-72.

WiLson, E.O. 1997. Biodiversidade. Rio de Janeiro, Nova Fronteira, 680p. 